\title{
MINIMIZAÇÃO DO CUSTO LOGÍSTICO DE FRETE NA DRISTRIBUIÇÃO DE ALIMENTOS UTILIZANDO PROGRAMAÇÃO LINEAR
}

\author{
Nathalia Tessari Moraes (Universidade De Caxias Do Sul - UCS) ntmoraes1@ucs.br \\ Bruna Gabriele de Matos (Universidade De Caxias Do Sul) bgmatos@ucs.br \\ Rafaela Boeira Cechin (Universidade de Caxias do Sul) rbcechin@ucs.br \\ Bruna Caroline Orlandin (Universidade De Caxias Do Sul - UCS) bcorlandin@ucs.br \\ Leandro Luís Corso (Universidade De Caxias Do Sul - UCS) 1lcorso@ucs.br
}

\section{Resumo}

A Programação Linear, como uma das técnicas da Pesquisa Operacional, firma-se cada vez mais como uma técnica que pode auxiliar as organizações na alocação dos seus recursos. Com o uso da modelagem matemática é possível representar problemas de logística e buscar uma minimização dos custos, por meio de um resultado ótimo. No estudo aqui apresentado, tem-se o caso de uma indústria de alimentos que contrata fretadoras para distribuir os seus produtos, busca-se então otimizar a escolha da fretadora adequada para cada demanda, considerando capacidade, custo e localização. Para isso, delimitou-se a função objetivo de minimização dos custos referentes a este serviço, e todas as variáveis e restrições, para que, com a utilização da ferramenta Solver, integrada ao Excel, fosse possível encontrar um resultado ótimo. Considerando um cenário atual onde as fretadoras são escolhidas sem nenhum método, conforme necessidades e depois comparando com o cenário proposto pela otimização foi possível perceber uma redução dos custos em apenas um mês analisado, no valor de R\$ 6.800,00, o que representa em torno de 7,43\% de redução dos custos, o que em longo prazo pode impactar diretamente nos resultados e na competitividade da empresa. Os resultados apresentados reforçam a importância de métodos assertivos para tomar decisões na logística das empresas, influenciando na lucratividade das mesmas. Além disso, o modelo aqui proposto pode servir para outras empresas que possuam um cenário semelhante.

Palavras-Chaves: Logística; Transporte; Programação linear; Frete.

\section{Introdução}

Para Moreira (2010) a Pesquisa Operacional (PO) lida com problemas de como conduzir e coordenar certas operações em uma organização, e tem sido aplicada a diversas áreas, incluindo indústria e transportes.

Considerando a importância da operação da logística nas empresas e os custos recorrentes de suas operações é importante que as empresas foquem em um dos objetivos da logística, de acordo com Christopher (2007), o de maximizar as lucratividades por meio da entrega de produtos com menor custo associado.

Com a utilização da Programação Linear (PL) é possível modelar os problemas de transporte e obter resultados otimizados. De acordo com Santos (2014), sempre que utiliza-se a 
modelagem para tomada de decisão, é necessária a criteriosa avaliação de cada conjunto de parâmetros buscando uma formulação correta do problema.

Neste estudo é apresentado uma indústria de alimentos que distribui seus produtos por meio da contratação de empresas que realizam fretes, no cenário atual não há nenhum método para a tomada de decisão das melhores formas de distribuir os seus produtos. Portanto, busca-se otimizar essa escolha, mostrando a melhor fretadora para cada demanda, considerando custos, capacidade e localização dos 5 clientes que a empresa entrega seus produtos que aqui serão analisados.

Contudo, para modelar matematicamente o problema apresentado, busca-se delimitar o objetivo, que é de minimizar os custos com fretes, além de definir as restrições e variáveis que caracterizam o sistema. Para que, por meio da PL, com o método Simplex, possa ser calculado um resultado ótimo para a escolha de fretadoras para o período de 4 semanas, correspondente a um mês de operação da indústria alimentícia, buscando melhores resultados.

\section{Pesquisa operacional}

Segundo Taha (2008) a Pesquisa Operacional surgiu ao longo da Segunda Guerra Mundial, na Inglaterra. Mello \& Mello (2003) salienta que a PO surgiu quando os Aliados se depararam com desafios de logística tática e estratégia militar, com grandes dimensões e alta complexidade e para isso, fizeram grupos multidisciplinares com engenheiros, matemáticos, físicos e cientistas das ciências sociais que aplicaram métodos científicos e criaram modelos matemáticos para resolver problemas.

Posteriormente Silva et al. (2010) define como a descrição de um sistema organizado com o auxílio de um modelo, e por meio da experimentação com o modelo, na descoberta da melhor maneira de operar o sistema, determinando melhores condições de aproveitamento dos recursos. Hillier \& Lieberman (2013) sintetizam um estudo de PO pelos seguintes passos: a definição do problema, coleta dos dados e formulação do modelo matemático para representar o problema.

Estes modelos que caracterizam o problema são classificados em função do tipo de equações e variáveis do modelo, como: Programação Linear, Programação Não-linear e Programação Inteira (GOLDBARG e LUNA, 2016).

\subsection{Programação linear}


A Programação Linear (PL) é tida com uma das técnicas de maior destaque na PO, devido a sua simplicidade na formulação de modelos matemáticos e resolução dos problemas, sendo bastante utilizada (GAMEIRO, ROCCO \& CAIXETA FILHO, 2011).

De acordo com Gonçalves \& Koprowinski (1995), a PL caracteriza-se como uma técnica de planejamento, a qual tem se constituído como uma das mais robustas. Pode colaborar com aspectos bastante procurados nas organizações, como no aumento dos lucros e diminuição dos custos. Colin (2007) considera como um meio de tratar o problema de alocação ótima de recursos escassos para a realização de atividades. Por ótimo é suposto que não haja outra solução que seja melhor do que a oferecida.

Segundo SILVA et al. (2010) os problemas de PL consideram as limitações dos recursos e representam algo a ser atingido. Podem ser resolvidos com algoritmos de solução, como o Solver, por exemplo (suplemento do software Excel).

Para HILLIER \& LIEBERMAN (2013), o modelo de PL deve conter as seguintes propriedades:

a) Proporcionalidade: refere-se à quantidade de recursos consumidos por uma dada atividade que devem ser proporcionais ao nível dessa atividade na solução final do problema.

b) Variáveis de decisão e parâmetros: deve ser sempre possível aperfeiçoar dada atividade em qualquer nível não negativo e qualquer razão de um dado recurso deve sempre ser utilizado;

c) Aditividade: o custo total é a soma dos componentes relacionados a cada atividade;

d) Separabilidade: deve-se reconhecer de forma separada o custo (ou uso de recursos) individual das operações de cada atividade.

Moreira (2007) reforça os principais elementos que existem em um modelo matemático:

a) Função Objetivo: é a solução do problema, mede o desempenho do mesmo, sendo uma expressão do que deseja-se minimizar ou maximizar, é formada por uma combinação linear das variáveis de decisão;

b) Variáveis de decisão e parâmetros: por meio da função-objetivo, surgem variáveis fundamentais, conhecidas como variáveis de decisão. Os parâmetros são valores fixos que 
fazem parte do problema;

c) Restrições: são expressas na forma de equações ou inequações matemáticas, representam os limites impostos sobre as ações com o objetivo de maximizar ou minimizar a função objetivo.

Tendo em vista que todo problema de PL pode ser representado por meio de uma função objetivo e de um conjunto de restrições, ambos lineares, na Equação 1 tem-se o modelo geral de PL (LOESCH; HEIN, 2009):

$$
\{\operatorname{Max}, \operatorname{Min}\} Z=c_{1} x_{1}+c_{2} x_{2}+\cdots+c_{n} x_{n}
$$

Sujeito a:

$$
\begin{gathered}
a_{11} x_{1}+a_{12} x_{2}+\cdots+a_{1 n} x_{n}\{=, \leq, \geq\} b_{1} \\
a_{21} x_{1}+a_{22} x_{2}+\cdots+a_{2 n} x_{n}\{=, \leq, \geq\} b_{2} \\
\cdot \\
\cdot \\
\cdot \\
a_{m 1} x_{1}+a_{m 2} x_{2}+\cdots+a_{m n} x_{n}\{=, \leq, \geq\} b_{m}
\end{gathered}
$$

Sendo:

$$
x_{1} \geq 0, x_{2} \geq 0, \ldots, x_{n} \geq 0
$$

Onde:

As variáveis $x_{1}, x_{2}, \ldots, x_{n}$, são estruturais do problema, escolhidas para que as restrições sejam satisfeitas e a função objetivo otimizada.

Os coeficientes $c_{1}, c_{2}, \ldots, c_{n}$ fazem parte da função objetivo e $a_{i j}$ e $b_{j}$ são os coeficientes das restrições, ou seja, dos limites a serem considerados.

A função objetivo mostra a meta que se deseja alcançar, pode ser de minimização $(\{\operatorname{Min} Z\}=\cdots)$ ou de maximização $(\{\operatorname{Max} Z\}=\cdots)$.

\subsection{Método Simplex}

Conforme LOESCH \& HEIN (2008), a aplicação do método Simplex na forma originalmente concebida exige que o problema esteja na forma padrão, ou seja, com suas variáveis de folga 
e/ou artificiais, com restrições de não-negatividade de todas variáveis e com valores da mão direita nas restrições não negativos.

Este método converte temporariamente todas as restrições de desigualdade em restrições de igualdade, somando uma nova variável a cada restrição "menor ou igual a" e subtraindo uma nova variável de cada restrição "maior ou igual a”. (RAGSDALE, 2009, p.164).

Para aplicação do método de PL pode-se utilizar o pacote de aplicativos do Microsoft Office Excel@ chamado Solver (HILLIER; LIEBERMAN, 2013) ou mesmo ferramentas como IBMCPLEX, Gurobi ou XPRESS Optimization.

\section{Metodologia}

Considerando-se o caso de uma indústria de alimentos que contrata fretadoras para realizar a distribuição de produtos, levando-os para cinco clientes diferentes em cidades próximas do Estado de Santa Catarina.

Para que a logística de distribuição dos alimentos ocorra da melhor forma, buscou-se minimizar os custos de logística referente ao frete, por meio da Programação Linear, uma das técnicas de PO, que apoiou na tomada de decisão da melhor fretadora para cada semana do período de 1 mês, considerando também a demanda, custo e localização.

No entanto, é necessário realizar a modelagem do problema, para isso seguiram-se os seguintes passos mostrados na Figura 1.

Figura 1- Fluxograma do problema
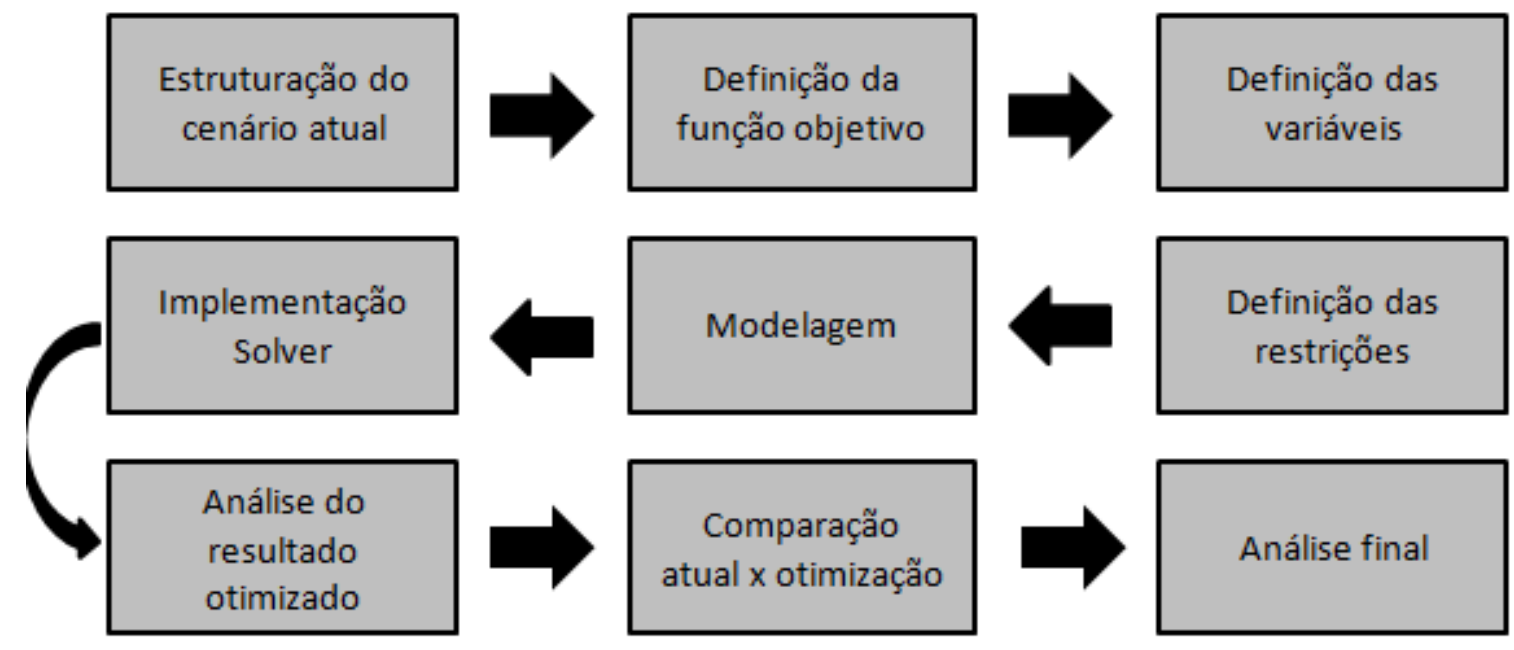

Fonte: Os autores (2020) 
Neste trabalho, para solucionar o problema de PL foi utilizada a ferramenta Solver, integrada ao software Microsoft Excel®.

\section{Resultados obtidos}

A indústria de alimentos contrata terceiros para distribuir seus produtos, consistindo em três fretadoras diferentes F1, F2 e F3, que levam a 5 clientes diferentes aqui analisados. Atualmente não há nenhum critério de escolha das fretadoras, não partiu de avaliações analíticas e nem simulações ou otimizações, foram contratadas conforme as necessidades com o decorrer do tempo.

É obedecido somente questões de custos, prazos e restrições de cada fretadora, sendo que os custos definem-se pela quantidade de caixas transportadas, sempre respeitando a capacidade máxima de cada fretadora.

Atualmente, as entregas dos produtos apresentam o seguinte cenário representado no Quadro 1.

Quadro 1- Cenário atual

\begin{tabular}{|l|c|c|c|c|}
\hline & \multicolumn{2}{|c|}{ Semana 1 } & \multicolumn{2}{c|}{ Semana 2 } \\
\hline & $\begin{array}{c}\text { Número de } \\
\text { Caixas }\end{array}$ & Fretadora & $\begin{array}{c}\text { Número de } \\
\text { Caixas }\end{array}$ & Fretadora \\
\hline Cliente 1 & 17 & F2 & 16 & F2 \\
\hline Cliente 2 & 20 & F1 & 19 & F1 \\
\hline Cliente 3 & 15 & F2 & 13 & F2 \\
\hline Cliente 4 & 14 & F1 & 16 & F2 \\
\hline Cliente 5 & 16 & F3 & 17 & F2 \\
\hline & Custo total & $\mathbf{2 4 . 1 8 0 , 0 0}$ & Custo total & $\mathbf{2 0 . 0 3 0 , 0 0}$ \\
\hline & \multicolumn{2}{|c|}{ Semana 3 } & Número de & Fretadora \\
\hline & Caixas & Fretadora & Caixas & F3 \\
\hline Cliente 1 & 12 & F1 & 15 & F3 \\
\hline Cliente 2 & 16 & F1 & 13 & F1 \\
\hline Cliente 3 & 20 & F2 & 18 & F2 \\
\hline Cliente 4 & 15 & F1 & 16 & F3 \\
\hline Cliente 5 & 14 & F2 & Custo total & $\mathbf{2 4 . 6 1 0 , 0 0}$ \\
\hline & Custo total & $\mathbf{2 2 . 8 7 0 , 0 0}$ & Fonte: Os autores (2020) & \\
\hline
\end{tabular}


No mês analisado o custo total da empresa com frete foi de $\mathrm{R} \$ 91.690,00$, visando minimizar este custo, foi utilizado a PL, para contribuir em uma melhor tomada de decisão na contratação dessas fretadoras.

Por tanto, se elencam as variáveis que representam o modelo, dessa forma, pode-se ver no Quadro 2 as variáveis e seus significados, onde, por exemplo, x1 é a quantidade de caixas enviadas para o cliente 1 pela fretadora 1 e assim por diante.

Quadro 2- Variáveis do problema

\begin{tabular}{|c|c|c|c|}
\hline $\begin{array}{c}\text { Ajusta o } \\
\text { tamanho das } \\
\text { colunas para } \\
\text { o texto ficar } \\
\text { numa linha }\end{array}$ & Fretadora 1 & Fretadora 2 & Fretadora 3 \\
\hline Cliente 1 & $\mathrm{x} 1$ & $\mathrm{x} 2$ & $\mathrm{x} 3$ \\
\hline Cliente 2 & $\mathrm{x} 4$ & $\mathrm{x} 5$ & $\mathrm{x} 6$ \\
\hline Cliente 3 & $\mathrm{x} 7$ & $\mathrm{x} 8$ & $\mathrm{x} 9$ \\
\hline Cliente 4 & $\mathrm{x} 10$ & $\mathrm{x} 11$ & $\mathrm{x} 12$ \\
\hline Cliente 5 & $\mathrm{x} 13$ & $\mathrm{x} 14$ & $\mathrm{x} 15$ \\
\hline
\end{tabular}

A função objetivo representa a função de minimização de custos, sendo que cada uma das variáveis é multiplicada pelo custo unitário da caixa cobrado pela fretadora para cada cliente, como segue:

\footnotetext{
$\operatorname{Min} \mathrm{f}(\mathrm{X})=200 * \mathrm{X}_{1}+180 * \mathrm{X}_{2}+270 * \mathrm{X}_{3}+360 * \mathrm{X}_{4}+500 * \mathrm{X} 5+480 * \mathrm{X}_{6}+240 * \mathrm{X}_{7}+220 * \mathrm{X}_{8}+400 * \mathrm{X}_{9}+$ $370 * \mathrm{X}_{10}+200 * \mathrm{X}_{11}+380 * \mathrm{X}_{12}+400 * \mathrm{X}_{13}+250 * \mathrm{X}_{14}+340 * \mathrm{X}_{15}$
}

Após definido o objetivo, definem-se as restrições do problema, que devem ser números nãonegativos e inteiros para este problema, considerando que a capacidade máxima de cada fretadora é de 20 caixas (tem divergência com o número a seguir). No entanto, as restrições podem ser assim representadas:

a) Número máximo de caixas enviadas por cada fretadora é de 20 unidades;

b) $\mathrm{x}_{1}+\mathrm{x}_{2}+\mathrm{x}_{3}=$ número de caixas que vai para o cliente 1 ;

c) $\mathrm{x}_{4}+\mathrm{x}_{5}+\mathrm{x}_{6}=$ número de caixas que vai para o cliente 2 ;

d) $\mathrm{x}_{7}+\mathrm{x}_{8}+\mathrm{x}_{9}=$ número de caixas que vai para o cliente 3 ;

e) $\mathrm{x}_{10}+\mathrm{x}_{11}+\mathrm{x}_{12}=$ número de caixas que vai para o cliente 4 ; 
f) $\mathrm{X}_{13}+\mathrm{x}_{14}+\mathrm{x}_{15}=$ número de caixas que vai para o cliente 5 ;

g) Todas variáveis possuem valor igual ou maior que zero;

h) Todas variáveis são números inteiros.

Por meio da modelagem matemática e com a aplicação da ferramenta Solver, foi possível otimizar a distribuição dos produtos da empresa, apresentando o menor custo. No Quadro 3 pode-se ver o resultado da otimização.

Quadro 3- Resultado da otimização

\begin{tabular}{|c|c|c|c|c|}
\hline & \multicolumn{2}{|c|}{ Semana 1} & \multicolumn{2}{|c|}{ Semana 2} \\
\hline & $\begin{array}{l}\text { Número de } \\
\text { Caixas }\end{array}$ & Fretadora & $\begin{array}{l}\text { Número de } \\
\text { Caixas }\end{array}$ & Fretadora \\
\hline Cliente 1 & 17 & $\mathrm{~F} 3$ & 16 & $\mathrm{~F} 1$ \\
\hline Cliente 2 & 20 & $\mathrm{~F} 2$ & $10 ; 9$ & $\mathrm{~F} 1 ; \mathrm{F} 3$ \\
\hline Cliente 3 & 15 & $\mathrm{~F} 1$ & 13 & F3 \\
\hline Cliente 4 & 14 & $\mathrm{~F} 2$ & $8 ; 8$ & F2;F3 \\
\hline \multirow[t]{4}{*}{ Cliente 5} & 16 & $\mathrm{~F} 2$ & $10 ; 7$ & F2;F3 \\
\hline & Custo total & \begin{tabular}{|l|}
$24.990,00$ \\
\end{tabular} & Custo total & $25.840,00$ \\
\hline & \multicolumn{2}{|c|}{ Semana 3} & \multicolumn{2}{|l|}{ Semana 4} \\
\hline & $\begin{array}{l}\text { Número de } \\
\text { Caixas }\end{array}$ & Fretadora & $\begin{array}{l}\text { Número de } \\
\text { Caixas }\end{array}$ & Fretadora \\
\hline Cliente 1 & $4 ; 6 ; 2$ & $\mathrm{~F} 1 ; \mathrm{F} 2 ; \mathrm{F} 3$ & 15 & $\mathrm{~F} 1$ \\
\hline Cliente 2 & $10 ; 6$ & $\mathrm{~F} 1 ; \mathrm{F} 3$ & $7 ; 6$ & $\mathrm{~F} 2 ; \mathrm{F} 3$ \\
\hline Cliente 3 & 20 & $\mathrm{~F} 2$ & $8 ; 10$ & $\mathrm{~F} 2 ; \mathrm{F} 3$ \\
\hline Cliente 4 & $10 ; 5$ & F2;F3 & 16 & $\mathrm{~F} 2$ \\
\hline \multirow[t]{2}{*}{ Cliente 5} & 20 & F2;F3 & $5 ; 10 ; 5$ & F2;F3 \\
\hline & Custo total & $21.420,00$ & Custo total & $12.640,00$ \\
\hline
\end{tabular}

Com a otimização o custo total do mês em frete seria de $\mathrm{R} \$ 84.890,00$.

Pode-se analisar a comparação do custo atual e do custo com otimização na Tabela 1.

Tabela 1- Comparação dos custos

\begin{tabular}{ccc}
\hline Semanas & Custo Atual & Custo proposto \\
\hline 1 & $24.180,00$ & $24.990,00$ \\
2 & $20.030,00$ & $25.840,00$ \\
3 & $22.870,00$ & $21.420,00$ \\
4 & $24.610,00$ & $12.640,00$
\end{tabular}




\begin{tabular}{ccc}
\hline Total & $\mathbf{9 1 . 6 9 0 , 0 0}$ & $\mathbf{8 4 . 8 9 0 , 0 0}$ \\
\hline
\end{tabular}

Fonte: Os autores (2019)

Após comparação, percebe-se a eficácia do método implementado, que apresenta uma redução de $\mathrm{R} \$ 6.800,00$ no custo com a contratação de fretadoras em apenas um mês, impactando diretamente no lucro da empresa.

\section{Considerações finais}

Por meio da modelagem matemática e das técnicas de PO foi possível entender o problema do caso apresentado e resolvê-lo, apresentado uma escolha melhor na contratação de fretadoras para distribuir os produtos da indústria de alimentos estudada. Após, comparou-se o custo atual com os custos que apresentará se for adotada a otimização realizada.

No entanto, nas 4 semanas do mês que foram analisadas, percebeu-se uma redução de R\$ $6.800,00$, que representa em torno de $7,43 \%$ de minimização dos custos em fretes, somente em um mês.

O resultado pode ser bastante expressivo para uma empresa de pequeno porte e para um período curto de tempo de somente um mês, que no decorrer de um período maior pode apresentar uma redução de custo significativa, impactando diretamente no lucro da empresa. Apesar de a técnica não ser inovadora, traz ganhos significativos para as empresas, considerando que melhorando a logística de distribuição dos seus produtos e com consequente redução de custos gera-se uma margem maior para novos investimentos, gerando melhor competitividade.

Portanto, este estudo fortalece a validade da PL, como uma das técnicas de PO, para melhorar a tomada de decisão nas organizações e proporcionar melhores resultados, além de servir como base para outras empresas que possuam um cenário próximo a este aqui apresentado. 


\section{REFERÊNCIAS}

CHRISTOPHER, M. Logística e gerenciamento da cadeia de suprimentos. São Paulo: Thomson, 2007.

COLIN, Emerson C. Pesquisa Operacional. Editora: LTC, 2007.

GAMEIRO, A. H., ROCCO, C. D., \& CAIXETAFILHO, J. V. Modelo matemático para otimização e avaliação de unidade produtora de leite caracterizada pela integração lavoura-pecuária: consideração de parâmetros econômicos, logísticos e ambientais. In CONGRESSO DA SOCIEDADE BRASILEIRA DE ADMINISTRAÇÃO E SOCIOLOGIA RURAL, 49, Belo Horizonte, Anais, SOBER, 2011.

GOLDBARG, M. C. \& LUNA, H. P. L. Programação linear fluxos em redes. 1 ed., Rio de Janeiro, Elsevier, 2016.

GONÇAlveS, A.; KOPROWINSKI, S. O. Pequena Empresa no Brasil. São Paulo: Imprensa Oficial do Estado, Editora da Universidade de São Paulo, 1995.

HILLIER, Frederick S.; LIEBERMAN, Gerald. J. Introdução à Pesquisa Operacional. 9º ed. São Paulo: Editora Bookman, 2013.

LOESCH, C.; HEIIN, N. Pesquisa Operacional - fundamentos e modelos. 1. ed. São Paulo: Saraiva, 2008.

MELLO, J. C. C. B.; MELLO, M. H. C. S. Integração entre o ensino de cálculo e o de pesquisa operacional. Revista Produção, v. 13, n. 2, p. 123, 2003.

MOREIRA, D. A. Pesquisa operacional: curso introdutório. São Paulo: Thomson Learning, p.356, 2007.

MOREIRA, D. A. Pesquisa Operacional: Curso Introdutório. São Paulo: Thomson Learning, 2010.

RAGSDALE, Clift T. Modelagem e Análise de Decisão. 6a edição. São Paulo: Cengage Learning, 2009.

SANTOS, Bruno Samways Dos. Programação De Ônibus Interurbano Utilizando A Técnica De Programação Linear Inteira. Dissertação de Mestrado em Engenharia de Produção - Universidade Tecnológica Federal do Paraná, Ponta Grossa, 2014.

SILVA, Ermes Medeiros da, SILVA, Elio Medeiros da, GONCALVES, Valter. Pesquisa operacional: programação linear, simulação. 3. ed. São Paulo : Atlas,2010.

TAHA, Hamdy A. Pesquisa operacional uma visão geral. São Paulo: Pearson Prentice Hall, 2008. 